\title{
Analysis of J-Pole Antenna Configurations for Underwater Communications
}

\author{
O. Aboderin, S. I. Inácio, H. M. Santos, M. R. Pereira, L. M. Pessoa, H. M. Salgado \\ INESC TEC and Faculdade de Engenharia, Universidade do Porto \\ Rua Dr. Roberto Frias, s/n \\ 4200-465 Porto, Portugal \\ (oluyomi.aboderin, mario.r.pereira, hugo.m.santos, luis.m.pessoa, hsalgado)@inesctec.pt, sofia.inacio@fe.up.pt,
}

\begin{abstract}
The capability of relatively high-speed short-range communications of Autonomous Underwater Vehicles (AUVs) in underwater scenarios, for example, for communication between vehicles or when the AUV is approaching a docking station for downloading of data gathered during a survey mission, is becoming a relevant application in the context of sea exploration and mining.

In this paper the analysis of the J-pole antenna and two of its configurations namely Super J-pole and Collinear J-pole antennas are presented, aimed at improving the propagation distance and data rates when such antennas are installed on AUV for onward usage in underwater communications. The performance of these three antennas is assessed through simulation in fresh and sea water, operating in the High Frequency (HF) band. These antennas are compared in terms of bandwidth and directivity which are important elements in the transmission and reception of electromagnetic signals.
\end{abstract}

The results obtained show that these antennas will be desirable both for improved data rates and propagation distance in fresh and sea water. The antennas were designed with FEKO electromagnetic simulation software.

Keywords-Autonomous Underwater Vehicle, antennas, J-pole, underwater communications, directivity, bandwidth.

\section{INTRODUCTION}

Research in the field of underwater communications continues to generate a lot of interest worldwide, due to a wide range of applications that this technology covers, which include: offshore oil and gas field monitoring, coastline protection and surveillance, oceanographic data collection which will require data exchange between two or more Autonomous Underwater Vehicles (AUVs) and underwater environmental observation for exploration [1, 2]. It is also a known fact that acoustic and ultrasonic systems have been a standard technology for underwater communications as they are typically good for long range communications (up to tens of kilometers). But this technology is unfit for real-time and broadband underwater wireless sensor networks because of high latency, poor immunity to noise and low data-rates $[1,3$, 4]. Another technology that has been considered for underwater application is optical wireless communication, which allows high data-rates and low latency, which are significant advantages over acoustic systems. However, these technologies require very good alignment and are also affected by marine fouling and suspended particles in water [3-5]. The third technology in line for underwater communication is electromagnetic (EM) communication [6]. The initial research in the use of this technology for underwater was carried out in the 60 s, with only a few practical work done, due to the inherently limitation associated with the huge attenuation of EM waves in water at high frequencies. In recent years it has been proven that EM underwater systems operating in the HF, band can benefit from the reduced attenuation in this band, and are able to deliver data rates of up to $10 \mathrm{Mbps}$ at short range, with the added advantage of not being affected by dirty water and not requiring strong alignment. These properties, makes EM interesting for certain underwater applications scenarios. Based on these analysis, performance evaluation of the J-pole antenna and two of its configurations that has higher advantages over the J-pole in terms of bandwidth and gain in air have been designed, simulated and compared in terms of maximum directivity and bandwidth in fresh and sea water scenarios.

J-pole typically resembles the shape of letter "J," with a half-wave vertical element end-fed by a quarter-wave matching stub. The addition of a phasing stub and half wavelength vertical element to ordinary J-pole yields super J-pole antennas and when this phasing stub is replaced with a phasing coil, another configuration of J-pole named collinear J-pole results. In air, it has been shown that super $\mathbf{J}$ antennas have higher bandwidth and higher gain than the J-pole antenna [7] and the 
collinear $\mathbf{J}$ antenna improves further on the performance of super $\mathbf{J}$ antenna [8]. It is in this regards that this work considers the analysis of the J-pole antennas and its configurations in underwater (fresh and sea) applications. It is to be noted that to the best of the authors' knowledge these antennas have only been designed and fabricated for usage in air which means the previously recorded analysis only considered their respective usage in air or for airborne operations. Thus, the J-pole, super$\mathbf{J}$ and collinear $\mathbf{J}$ antennas have been extensively investigated for fresh and sea water scenarios operating at $40 \mathrm{MHz}$ in the media. The aim is to consider their possible use in AUVs or Remote Operated Vehicles (ROVs) in fresh and sea water operations.

The rest of this paper is organised as follows: section II presents the propagation of electromagnetic waves in the media; in section III the design of the underwater antennas is presented with two subsections dedicated to the design for usage in fresh water and for sea water respectively; simulation results and interpretations are given in section IV, and the conclusions are addressed in section $\mathrm{V}$.

\section{Propagation OF EleCtromagnetic WAVES IN THE MEDIA}

Designing of antennas for underwater applications requires thorough understanding of the effect of the conductivity, $\sigma$, on the antenna element when the propagating medium is fresh or sea water. For instance, in fresh water conductivity ranges from $0.005 \mathrm{~S} / \mathrm{m}$ to $0.05 \mathrm{~S} / \mathrm{m}$ [9], whereas the value in sea water is 4 $\mathrm{S} / \mathrm{m}$. EM propagation in any medium is described by its propagation constant, $\gamma$, which is given as

$$
\gamma=\sqrt{j \omega \mu(j \omega \varepsilon+\sigma)}=\alpha+j \beta
$$

where $\omega$ is the angular frequency $(\mathrm{rad} / \mathrm{s}), \mu$ is the magnetic permeability, which is equal to the free space permeability, $\varepsilon$ is the dielectric permittivity in water (relative permittivity of 81). The propagating medium can be classified as a dielectric media or insulator when $\sigma / \omega \varepsilon<<1$, which means the displacement current is much greater than the conductive current. Conversely, when $\sigma / \omega \varepsilon \gg 1$, the media is considered good conductor, meaning the conductive current is much greater than the displacement current [10]. Based on this, the dependence of $\sigma / \omega \varepsilon$ as a function of operating frequency for fresh and sea water is shown in Fig. 1. As seen here, fresh water behaves as a conductor for frequencies below $10 \mathrm{MHz}$, thereafter behaves as a dielectric media, whereas the transition from conducting to dielectric media occurs at $888 \mathrm{MHz}$ in sea water. This implies that for lower frequencies, sea water behaves as a good conductor.

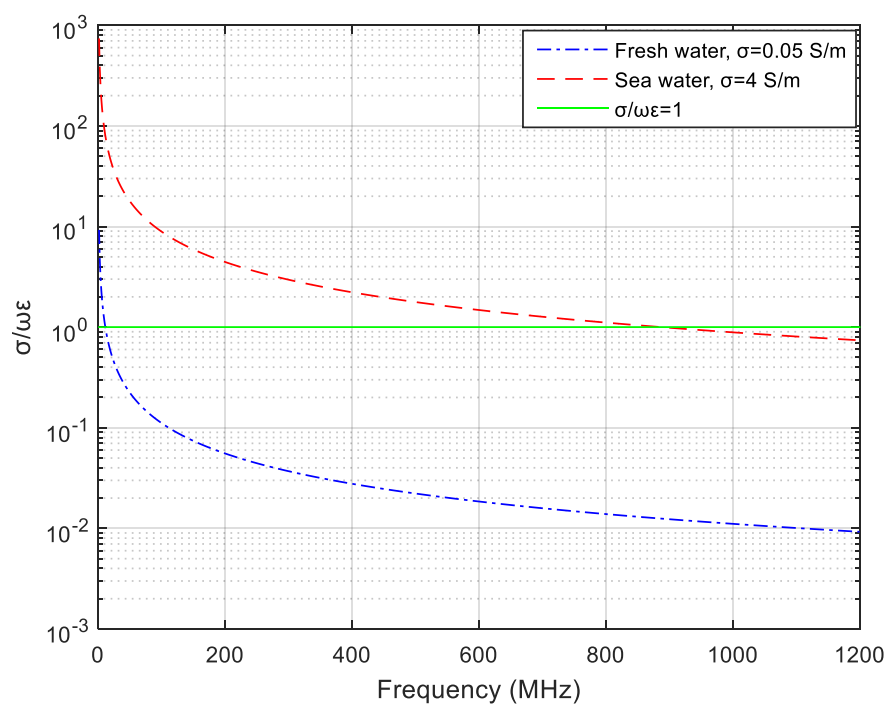

Fig.1: Attenuation of an electromagnetic wave propagating in fresh and sea water

In equation (1) the real part, alpha, is the attenuation constant $(\mathrm{Np} / \mathrm{m})$ and the imaginary part is the phase constant $(\mathrm{rad} / \mathrm{m})$. This can be written as $[1,11]$.

$$
\begin{aligned}
& \alpha=\omega \sqrt{\mu \varepsilon} \sqrt{\frac{1}{2}\left(\sqrt{1+(\sigma / \omega \varepsilon)^{2}}-1\right)} \\
& \beta=\omega \sqrt{\mu \varepsilon} \sqrt{\frac{1}{2}\left(\sqrt{1+(\sigma / \omega \varepsilon)^{2}}+1\right)}
\end{aligned}
$$

The attenuation of an electromagnetic waves, obtained from equation (2) is plotted in Fig. 2, as a function of frequency. Where it is observed that attenuation is relatively lower at frequencies up to hundredths of Mega Hertz in freshwater. Yet, in the sea water, the attenuation increase with increasing frequency. Based on this analysis, the antennas are designed to operate at lower frequency, which is important in order to get reasonable communication range especially in seawater. 


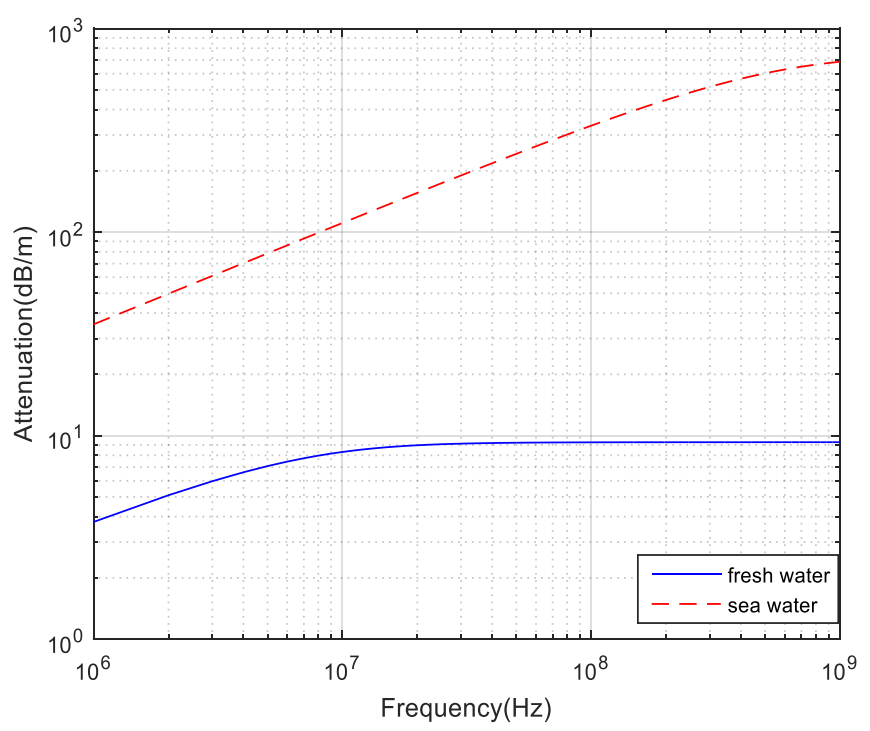

Fig.2. Behavior of $\sigma / \omega$ as a function of frequency in fresh and sea water [12]

\section{DESIGNING OF THE UNDERWATER ANTENNAS}

\section{A. Fresh Water}

The antennas in focus are the J-pole, collinear J-pole and super J-pole antennas. One other configuration of J-pole antenna which is not considered in this work is Slim Jim antenna which, unlike the super J-pole and the collinear J-pole, does not have any performance advantage over the J-pole antenna [7]. The antennas are designed using a thick copper wire of radius $1.5 \mathrm{~mm}$, covered by an insulator with thickness of $50 \mu \mathrm{m}$, and having a relative permittivity of 3 . Given that the wavelength is given by $\lambda=2 \pi / \beta$, the operation wavelength of the antennas, hence their dimensions, is dependent on both the permittivity and the conductivity of the medium. The antennas are designed using FEKO software and the models are presented in Fig. 3.

The dimensions of the antennas are presented in Table I. In the table, L1 is the stub base of the antennas and this is the same for the three antennas; L2 is their respective quarter wavelength stub or simply short arm; L3 is their half wavelength element or long arm and L4 is the length of the phasing coil. In the super J-pole, the stub (stub base and quarter wavelength long) and half wavelength element added are of the same length, while in the collinear J-pole antenna, the phasing stub is replaced by a $0.2 \lambda$ phasing coil. The dimension of the phasing coil is calculated based the on analysis of helical antennas as specified in $[13,14]$.

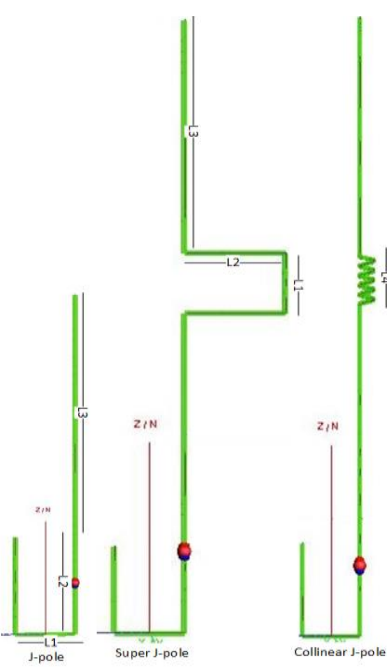

Fig. 3. Models of the J-pole, Super J-pole and Collinear Jpole antennas in underwater scenarios

TABLE I. DIMENSION OF THE ANTENNAS IN FRESH WATER

\begin{tabular}{|c|c|c|c|c|c|}
\hline S/No. & Antennas & $\mathbf{L 1}(\mathbf{m m})$ & $\mathbf{L 2} \mathbf{( m m})$ & $\mathbf{L 3}(\mathbf{m m})$ & $\mathbf{L 4}(\mathbf{m m})$ \\
\hline 1. & J-pole & 80.00 & 161.13 & 402.26 & - \\
\hline 2. & Super J-pole & 80.00 & 115.00 & 310.00 & - \\
\hline 3. & Collinear J-pole & 80.00 & 135.00 & 350.00 & 70.00 \\
\hline
\end{tabular}

\section{B. Sea Water}

In this medium the conductivity plays an important role in determining the dimensions of the antennas. The calculated wavelength at the same operating frequency of $40 \mathrm{MHz}$ is about one-quarter when compared with the one calculated in fresh water, taking a value of $244.44 \mathrm{~mm}$. The dimensions of the antennas are as presented in Table II.

TABLE II. Dimension of THE ANTENNAS IN SEA WATER

\begin{tabular}{|c|c|c|c|c|c|}
\hline S/No. & Antennas & $\mathbf{L 1}(\mathbf{m m})$ & $\mathbf{L 2} \mathbf{( m m )}$ & $\mathbf{L 3} \mathbf{( m m )}$ & $\mathbf{L 4}(\mathbf{m m})$ \\
\hline 1. & J-pole & 80.00 & 73.00 & 299.00 & - \\
\hline 2. & Super J-pole & 80.00 & 49.00 & 178.00 & - \\
\hline 3. & Collinear J-pole & 80.00 & 50.00 & 180.00 & 36.00 \\
\hline
\end{tabular}




\section{SimUlATION RESUlTS AND INTERPRETATION}

\section{A. Fresh Water}

The antennas are simulated with the ports represented in Fig. 3 by the dot located in along the long arm. Fig. 4 presents the results of reflection coefficient, when the input impedance of the antenna is matched to the source. Indeed, the antennas were simulated by running various parametric analysis, during which the ports are made to slide between the quarter wavelength stub and the half wavelength elements. Through this method, the antennas were successfully matched, thus matching circuit are not needed for the operation. However at their respective input impedances, the super J-pole and collinear J-pole antennas have inductive imaginary parts which must be cancelled. Based on this, capacitors are added to cancel the inductive load for the two antennas. The value of the capacitors calculated and included for this purpose are $0.57 \mathrm{nF}$ and 0.29 $\mathrm{nF}$ for the super J-pole and collinear J-pole, respectively. Thus, the corresponding simulation results for the bandwidth (from the reflection coefficient measured at $-10 \mathrm{~dB}$ ) and directivity are given in Table III. The three antennas achieved relatively same bandwidth, with J-pole slightly higher than the other two antennas. In terms of directivity, the collinear J-pole and the super J-pole have higher directivity than the J-pole antenna. These are very encouraging results when considering usage of these antennas in AUVs as the achievable bandwidth and directivity are higher than what is obtained for when the loop and the dipole antennas [12].

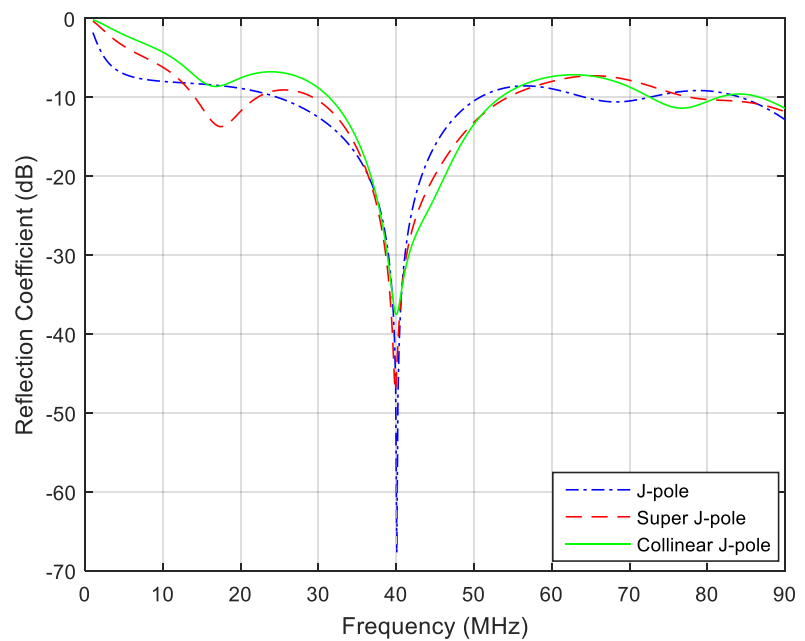

Fig. 4 Reflection coefficient against frequency for the antennas in fresh water

TABLE III.
\begin{tabular}{|c|c|c|c|c|}
\hline S/No. & Parameters & J-pole & $\begin{array}{c}\text { Super } \\
\text { J-pole }\end{array}$ & $\begin{array}{c}\text { Collinear } \\
\text { J-Pole }\end{array}$ \\
\hline 1. & Bandwidth (MHz) & 25.82 & 24.96 & 23.56 \\
\hline 2. & Max. Directivity (dB) & 4.65 & 7.13 & 8.02 \\
\hline
\end{tabular}

The simple circuit showing the addition of the capacitor as calculated, to cancel the inductive load is presented in Fig. 5 (a) and (b) for the super J-pole and the collinear J-pole antennas respectively. Also in water it is not possible to measure the farfields from an antenna, therefore the near-field equivalent was plotted. The normalized radiation pattern of the three antennas in the E-plane and H-plane are presented in Fig. 6 (a) and (b), respectively. In this medium, the J-pole antenna has wider beam than the other antennas.

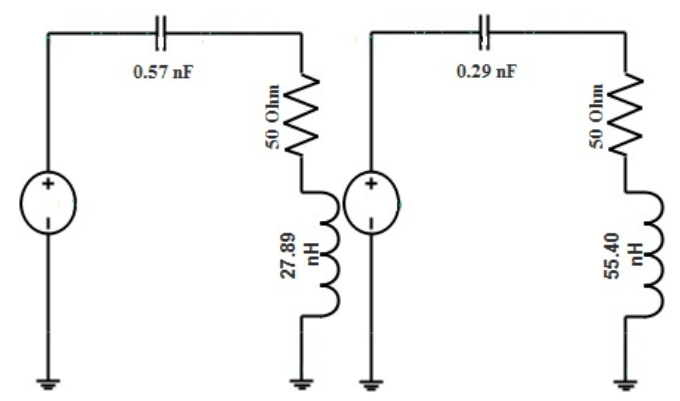

(a)

(b)

Fig. 5 (a) \& (b) Circuits showing capacitors added to cancel inductive load for super and collinear J-pole antennas respectively

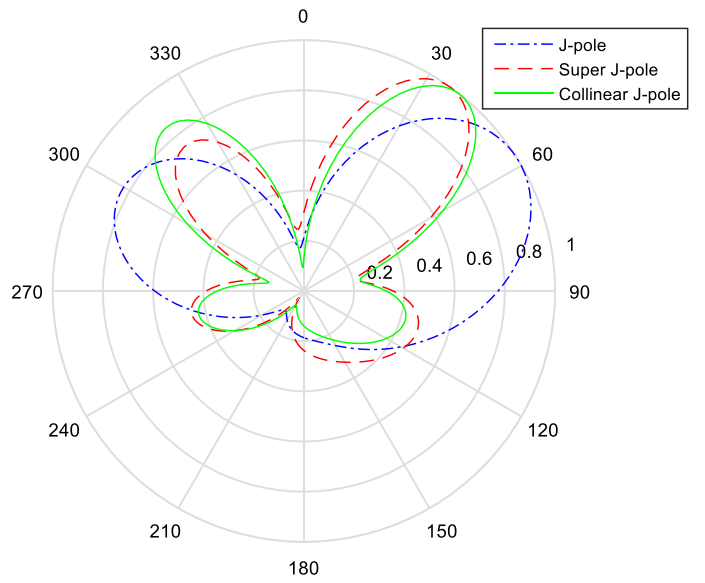

(a) 


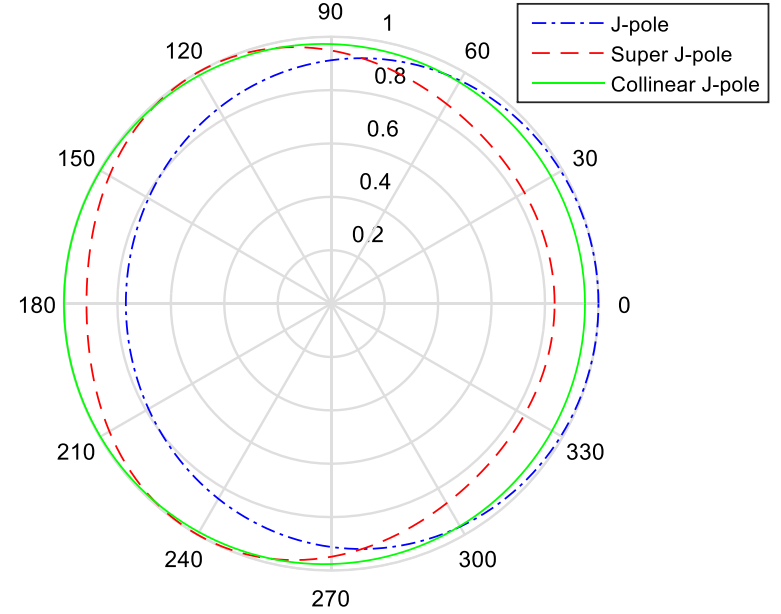

(b)

Fig. 6 (a) E-plane and (b) H-plane of the three antennas in fresh water

\section{B. Sea Water}

Simulation results corresponding to the bandwidth, input impedance and directivity characteristics of the antennas in this medium are presented in Table IV. Similarly, the results of the reflection coefficient is shown in Fig. 7. The input impedance of the antennas is very low in this medium due to change in the conductivity, and unlike fresh water, changing the ports locations for the antennas or sliding along the long arm does not improve the impedance in this medium. After several analysis of the antennas, the ports were located at 5\% distance along the long arms of the three antennas. With their respective input impedances, it simply means that matching circuits are important for the three antennas. To this purpose, Advance Simulation System (ADS) software was used to design matching circuits and to calculate the corresponding lumped elements, which were optimized for maximum performance. The matching circuit of the J-pole antenna is presented in Fig. 8 and similar circuits were designed for the super J-pole and the collinear J-pole antennas. The three antennas therefore exhibits a bandwidth that is closer to $30 \mathrm{MHz}$ in this medium. This is an important results considering that often underwater applications are carried out in salt water than fresh water. The radiation pattern of the antennas in sea water is shown in Fig. 9. The super J-pole antenna has the highest maximum directivity in this medium, followed by the collinear J-pole and then the J-pole antenna in that order. It is seen that due to the conductivity the antennas become more directive than when operating in fresh water.
TABLE IV. SimUlation RESUlts OF THE ANTENNAS IN SEA WATER

\begin{tabular}{|c|c|c|c|c|}
\hline S/No. & Parameters & J-pole & $\begin{array}{c}\text { Super } \\
\text { J-pole }\end{array}$ & $\begin{array}{c}\text { Collinear } \\
\text { J-Pole }\end{array}$ \\
\hline 1. & Bandwidth (MHz) & 27.92 & 28.32 & 28.45 \\
\hline 2. & Max. Directivity (dB) & 14.05 & 25.81 & 19.52 \\
\hline 3. & Input Impedance & 16.70 & 13.70 & 14.50 \\
\hline
\end{tabular}



Fig. 7 Reflection coefficient against frequency for the antennas in sea water



Fig. 8 Matching circuit for the antennas in sea water 




(a)



(b)

Fig. 9 (a) E-plane and (b) H-plane of the three antennas in sea water

Both the E-plane and the H-plane confirm that the three antennas radiated mainly in one direction in this medium. This is an important advantage over the loop and the dipole antennas that are bidirectional when simulated in this medium [12]. The implication of this is that the three antennas radiates all their respective powers in one direction only. In terms of beam, the J-pole antenna has wider beam than the other two antennas in the two media. Comparing these results with those of fresh water, there are noticeable difference seen in the radiation pattern in this medium due to the effect of conductivity earlier explained in section II, as the medium behaves like a good conductor.

\section{CONCLUSION}

An analysis of the performance characteristics of the J-pole, super J-pole and collinear J-pole antennas has been investigated in this work, with focus on the bandwidth and the directivity, which are two important parameters when considering data rates and propagation distance for underwater antennas. The results obtained show that the three antennas can be used in both fresh and sea water since they exhibit a bandwidth in the range of $23-30 \mathrm{MHz}$ in the media. In terms of directivity, the super J-pole and collinear J-pole antennas yield higher values which makes them more desirable for improved propagation distance.

Apart from this, like in air, changing the position of the ports can be used to balance the antennas in fresh water, however this is no longer the case in sea water.

Finally it is seen that the conductivity of the medium has great impact on the radiation pattern, namely its orientation. These are relevant results that need to be taken into consideration at the design stage of the antennas, as it is not obvious how directivity and the orientation of the radiation pattern will be affected by the properties of a conductive medium.

\section{ACKNOWLEDGEMENT}

This work has been developed as part of the project ENDUREEnabling Long Term Deployments of Underwater Robotic Platforms in Remote Oceanic Locations, under the EEA Grant No. PT02-Aviso4-0015, and TECSEA research infrastructure.

\section{REFERENCES}

[1] S. Jiang and S. Georgakopoulos, "Electromagnetic Wave Propagation into fresh water," presented at the International Conference on Detection and Classification of Underwater Targets, France, 2012. 
H. F. G. Mendez, F. Le Pennec, C. Gac, C. Person, and leee, "High Performance Underwater UHF Radio Antenna Development," 2011 IEEE Oceans Spain, 20112011.

[3] J. H. Goh, A. Shaw, and A. I. Al-Shamma'a, "Underwater Wireless Communication System," Journal of Physics, p. 6, 2009.

[4] H. Zhang, D. Geng, G. Zhang, and T. A. Gulliver, "The Impact of Antenna Design and Frequency on Underwater Wireless Communications," 2011 leee Pacific Rim Conference on Communications, Computers and Signal Processing (Pacrim), pp. 868-872, 20112011.

[5] X. Che, I. Wells, G. Dickers, and P. Kear, "TDMA frame design for a prototype underwater RF communication network," Ad Hoc Networks, vol. 10, pp. 317-327, May 2012.

[6] J. Lloret, S. Sendra, M. Ardid, and J. J. P. C. Rodrigues, "Underwater Wireless Sensor Communications in the $2.4 \mathrm{GHz}$ ISM Frequency Band," Sensors, vol. 12, pp. 4237-4264, Apr 2012.

[7] J. S. Huggins. (2012, 05/05). Improving the Super J-Pole Antenna. Available: http://www.hamradio.me/antennas/improvingthe-super-j.html
[8] S. Cerwin, Mobile and Maritime Antennas - The Super-J Maritime Antenna 21 ed. Straw, Dean. ARRL Antenna Book, 2007.

[9] L. V. B. Butler, "Underwater Radio Communication," Amateur Radio, p. 8, 1987.

[10] K. C. David, "Field and Wave Electromagnetics," IEE Proceedings-a-Science Measurement and Technology, vol. 131, pp. 660-660, 19841984.

[11] K. P. Hunt, J. J. Niemeier, and A. Kruger, "Short Paper: Antennas for Mussel-Based Underwater Biological Sensor Networks in Rivers," presented at the The International Conference on UnderWater Networks and Systems (WUWNet), Woods Hole, MA, USA, 2010.

[12] S. I. Inacio, M. R. Pereira, H. M. Santos, L. M. Pessoa, F. B. Teixeira, M. J. Lopes, et al., "Antenna design for underwater radio communications," presented at the OCEAN, Shanghai, China, 2016.

[13] A. R. Djordjevic, A. G. Zajic, M. M. Ilic, and G. L. Stuber, "Optimization of helical antennas," IEEE Antennas and Propagation Magazine, vol. 48, pp. 107-115, Dec 2006.

[14] C. A. Balanis, Antenna Theory Analysis and Design, 3rd Edition ed. New Jersey, USA: John Wiley \& Sons, 2005. 